\title{
High performance liquid level monitoring system based on polymer fiber Bragg gratings embedded in silicone rubber diaphragms
}

\author{
Carlos A. F. Marques*a, Gang-Ding Peng ${ }^{\mathrm{b}}$, David J. Webb ${ }^{\mathrm{a}}$ \\ ${ }^{a}$ Aston Institute of Photonic Technologies, Aston University, B4 7ET, United Kingdom \\ ${ }^{\mathrm{b}} \mathrm{School}$ of Electrical Engineering and Telecommunications, University of New South Wales, \\ Sydney NSW 2052, Australia
}

\begin{abstract}
Liquid-level sensing technologies have attracted great prominence, because such measurements are essential to industrial applications, such as fuel storage, flood warning and in the biochemical industry. Traditional liquid level sensors are based on electromechanical techniques; however they suffer from intrinsic safety concerns in explosive environments. In recent years, given that optical fiber sensors have lots of well-established advantages such as high accuracy, costeffectiveness, compact size, and ease of multiplexing, several optical fiber liquid level sensors have been investigated which are based on different operating principles such as side-polishing the cladding and a portion of core, using a spiral side-emitting optical fiber or using silica fiber gratings. The present work proposes a novel and highly sensitive liquid level sensor making use of polymer optical fiber Bragg gratings (POFBGs). The key elements of the system are a set of POFBGs embedded in silicone rubber diaphragms. This is a new development building on the idea of determining liquid level by measuring the pressure at the bottom of a liquid container, however it has a number of critical advantages. The system features several FBG-based pressure sensors as described above placed at different depths. Any sensor above the surface of the liquid will read the same ambient pressure. Sensors below the surface of the liquid will read pressures that increase linearly with depth. The position of the liquid surface can therefore be approximately identified as lying between the first sensor to read an above-ambient pressure and the next higher sensor. This level of precision would not in general be sufficient for most liquid level monitoring applications; however a much more precise determination of liquid level can be made by linear regression to the pressure readings from the sub-surface sensors. There are numerous advantages to this multi-sensor approach. First, the use of linear regression using multiple sensors is inherently more accurate than using a single pressure reading to estimate depth. Second, common mode temperature induced wavelength shifts in the individual sensors are automatically compensated. Thirdly, temperature induced changes in the sensor pressure sensitivity are also compensated. Fourthly, the approach provides the possibility to detect and compensate for malfunctioning sensors. Finally, the system is immune to changes in the density of the monitored fluid and even to changes in the effective force of gravity, as might be obtained in an aerospace application. The performance of an individual sensor was characterized and displays a sensitivity $(54 \mathrm{pm} / \mathrm{cm})$, enhanced by more than a factor of 2 when compared to a sensor head configuration based on a silica FBG published in the literature, resulting from the much lower elastic modulus of POF. Furthermore, the temperature/humidity behavior and measurement resolution were also studied in detail. The proposed configuration also displays a highly linear response, high resolution and good repeatability. The results suggest the new configuration can be a useful tool in many different applications, such as aircraft fuel monitoring, and biochemical and environmental sensing, where accuracy and stability are fundamental.
\end{abstract}

Keywords: Fiber Bragg gratings; Polymer optical fiber sensors; Liquid level monitoring systems.

\section{INTRODUCTION}

There are many measurement technologies to monitoring liquid levels, which are crucial to industrial applications, such as fuel storage, for providing flood warning and tides, and in the biochemical industry. Up to now, traditional liquid level sensors are based on electromechanical techniques; however they suffer from intrinsic safety concerns in explosive environments. Optical technologies like optical fiber sensors can give a better solution than traditional sensors. They are intrinsically safe in nature with no risk of explosion even under malfunction operation. A wide range of optical fiber liquid level sensor systems have been reported and they are based on different operating principles [1-4]. Optical fiber sensors using gratings in silica fiber [5-10] for liquid level applications have received great attention due to their wavelength response, linear output, sensitivity response, large dynamic range and compatibility with optical fiber

*c.marques@aston.ac.uk; phone +44 (0)121 204 3524; http://www1.aston.ac.uk/eas/research/groups/photonics/

\footnotetext{
Micro-structured and Specialty Optical Fibres IV, edited by Kyriacos Kalli,

Jiri Kanka, Alexis Mendez, Proc. of SPIE Vol. 9507, 95070N · ( 2015

SPIE - CCC code: 0277-786X/15/\$18 - doi: 10.1117/12.2180563
}

Proc. of SPIE Vol. 9507 95070N-1 
networks. However, all these sensors exhibit some drawbacks such as low sensitivity [10,11], limited range [13,17,18], long-term instability [12], limited resolution [9,10], high cost [9] and complicated manufacturing $[9,13,14,16]$.

On the other hand, polymer optical fiber Bragg gratings (POFBGs) are increasingly considered as a potential alternative to FBGs in silica fiber in certain sensing applications [11-13]. In POFs technology, there are a number of potential advantages, such as excellent flexibility, higher mechanical resistance to impacts and vibrations, low cost, and ruggedness. They can survive higher strain than silica and they possess a much lower Young's modulus. In view of these qualities, they have been used to measurements of temperature, humidity, strain, refractive index, acceleration and so on [11-15].

In this paper, a simple and highly sensitive liquid level sensor using POFBGs is investigated. The key element of the sensor is the POFBG embedded in a silicone rubber diaphragm. The performance of this sensor is compared with a similar sensor with an FBG inscribed in silica fiber and exhibits a factor of 5 improvement in sensitivity, resulting from the much lower elastic modulus of POF compared to silica fiber. The proposed configuration also displays a highly linear response, high resolution and good repeatability. Furthermore, the temperature behavior and measurement resolution were also studied in detail. Also, a novel configuration involving multiple pressure sensors is proposed that offers advantages over the single sensor, particularly an insensitivity to the density of the liquid being monitored.

\section{OPERATIONAL PRINCIPLE}

If a diaphragm is sealed over an air cavity and immersed in a liquid, the external pressure will increase with the liquid level or depth. The external pressure $p_{h}$ at a given depth $h$ will be will be related to the internal atmospheric pressure $p_{a}$ according to

$$
p_{h}=p_{a}+\rho g h
$$

where $\rho$ is the liquid density $\left(\mathrm{kg} / \mathrm{m}^{3}\right), g$ is the gravitational acceleration $\left(\mathrm{m} / \mathrm{s}^{2}\right)$ and $h$ is the height of the liquid.

A diaphragm disk of diameter $2 r$ and thickness $t$ is deflected when there is an external pressure increase owing to increasing liquid level. This in turn causes strain to appear across the diaphragm disk, and with an FBG attached to, or embedded in the disk, this strain can be measured.

For a clamped circular diaphragm, the center deflection $\delta_{c}$, is given by the following equation as long as the material remains within the elastic region [16]:

$$
\delta_{c}=\frac{3}{16}\left(1-v^{2}\right) \frac{\Delta p r^{4}}{E t^{3}}
$$

where $v$ is Poisson's ratio, $r$ is the radius of the diaphragm, and $E$ is Young's modulus of the diaphragm. $\Delta p$ is the difference of the internal and external cavity pressures. If the cavity is sealed with an internal pressure of the atmospheric pressure $p_{a}$, and the diaphragm deflection is sufficiently small such that the internal atmospheric pressure $p_{a}$ remains essentially constant, we will have $\Delta p=\rho g h$. The maximum strain $\varepsilon_{\max }$, at the center of the diaphragm is a linear function of $\Delta p$, and is given [16] as:

$$
\varepsilon_{\max }=\frac{3}{8}(1-v) \frac{\Delta p r^{2}}{t^{2}}
$$

The resultant deformation of the diaphragm will change the physical fiber dimensions resulting in a change in the Bragg wavelength $\lambda_{B}$. The wavelength shift $\Delta \lambda_{B}$, caused by this deformation is given as [17]:

$$
\Delta \lambda_{B}=\lambda_{B}\left(1-\rho_{e}\right) \varepsilon_{\max }
$$

where $\lambda_{B}$ is the initial Bragg wavelength and $\rho_{e}$ is the photo-elastic coefficient of the fiber. By monitoring the wavelength shift of the FBG, the level of liquid can be inferred.

If we take typical values for silicone rubber [18] $(v=0.47, r=9.5 \mathrm{~mm}, t=1.1 \mathrm{~mm}$, and $E=0.0016 \mathrm{GPa}), \delta_{c}$ calculated from Eq. (2) is $4.1 \mathrm{~mm}$ for a water level of $75 \mathrm{~cm}$. 


\section{DESIGN AND FABRICATION OF SENSORS}

\subsection{POFBGs Fabrication and diaphragm preparation}

Several identical FBGs were inscribed in single-mode POF fabricated from PMMA - for details of the fabrication see [19]. The single-mode fiber has a core diameter of $\sim 8 \mu \mathrm{m}$ and an outer diameter of $\sim 125 \mu \mathrm{m} .10 \mathrm{~cm}$ long POF sections were laid in a v-groove and taped down using polyimide tape to prevent them moving during inscription, which was carried out by illuminating from above a phase mask placed on top of the POF using $325 \mathrm{~nm}$ UV light from a heliumcadmium (HeCd) laser with a power output of $30 \mathrm{~mW}$. The $\mathrm{HeCd}$ laser beam was focused vertically downward using a $10 \mathrm{~cm}$ focal length cylindrical lens, through the phase mask, and onto the fiber. A butt-coupled connection was made between one arm of a $1550 \mathrm{~nm}$ single-mode silica coupler and the POF using a fiber connector/angled physical contact (FC/APC) connector on the silica fiber. A small amount of index matching gel was used in order to reduce Fresnel reflections, lowering the background noise. The inscription process was monitored using a broadband light source (provided by Thorlabs ASE-FL7002-C4 centered at $1560 \mathrm{~nm}$ ), and an optical spectrum analyzer (OSA) connected to the coupler. The optimum inscription time for this fiber is between 35 and 45 min. Following grating inscription, the POF sections containing FBGs were UV-glued (Norland 78 ) to one $8^{\circ}$ angled silica fiber pigtail.

The silicone rubber used for the diaphragm was prepared by mixing homogeneously liquids of silicone rubber (SILASTIC $^{\circledR}$ T-4 base and SILASTIC ${ }^{\circledR}$ T-4 catalyst from Dow Corning Corporation) in a ratio of 100:10 by volume. The de-gassed silicone rubber solution was poured in a circular plastic container $50 \mathrm{~mm}$ in diameter and $1.1 \mathrm{~mm}$ in height, in which was also placed the POF containing the FBG. Additional care was taken to ensure the POFBG was at the center of the diaphragm. To guarantee a reasonable uniformity of the diaphragm, a metal piece was placed on top of the container to exert a slight load. Furthermore, the viscous liquid nature of the mixture before curing helps ensure the uniformity of the diaphragm. With regard to uniformity, diaphragms were obtained with thickness of $1.05 \mathrm{~mm}$. The mold was kept undisturbed for 24 hours at room temperature to allow the silicone rubber to set.

\subsection{Single POFBG sensor}

The sensor configuration is presented in Fig. 1 (a). It is based on an aluminum gasket, which houses the silicone rubber diaphragm with a POFBG embedded directly in it. The square aluminum gasket piece has a $50 \mathrm{~mm}$ width and $10 \mathrm{~mm}$ height, containing a central cavity $15 \mathrm{~mm}$ in diameter and with a depth of $5 \mathrm{~mm}$. The retaining ring had a $40 \mathrm{~mm}$ diameter and the central hole a $19 \mathrm{~mm}$ diameter. To fix the diaphragm in position, silicone sealant was placed around the rim of the diaphragm on both sides, and this effectively sandwiched the diaphragm between the base and retaining ring.

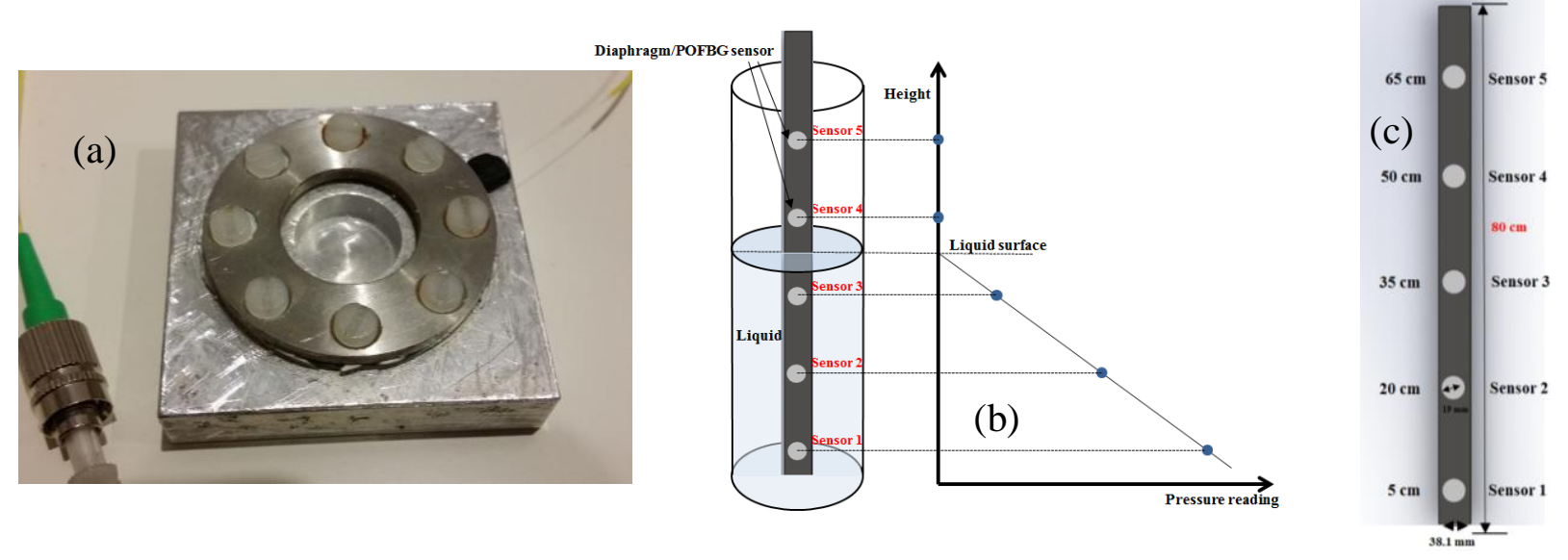

Fig. 1. (a) Design of the sensor system using a single POFBG. (b) Left: five discrete pressure sensors, with three submerged in liquid; right: determination of liquid level using linear regression. (c) Diagram of the acrylic tube sensor arrangement using multi-POFBGs.

\subsection{Multi-sensor based system}

This is a new development that builds on the idea of determining liquid level by measuring the pressure at the bottom of the liquid container (Fig. 1 (b)); but with some critical advantages as presented in the 'Abstract' section. The design of the prototype multiple sensor configuration consists of an acrylic tube $(80 \mathrm{~cm}$ length, with $3.2 \mathrm{~mm}$ wall thickness and 
$38.1 \mathrm{~mm}$ outside dimension), with windows drilled at equidistant positions along it as shown in Fig. 1 (c). It contains five sensors positioned over $15 \mathrm{~mm}$ diameter holes spatially separated by $150 \mathrm{~mm}$ (with the first hole separated by $50 \mathrm{~mm}$ from the tube base). The sensors were then placed at positions aligned with the window positions such that the FBG center aligned with the window center. As in the single sensor configuration, a silicone sealant was used to seal the sensing area.

\section{MODELING ANALYSIS}

To verify the proposed configuration and structure, the diaphragm/POFBG sensors are analysed by simulation using a finite element method (SolidWorks software). The parameters of the sensor in the following calculation and simulation are the same as those in the above 'operational principle' section. A standard mesh was used with total nodes of 447024 and total elements of 240980 (see Fig. 2 (a)). Fixture geometries were applied to acrylic tube and external loads (pressure variation and gravity) were distributed over the measurement area (diaphragm). The finite element method provides a powerful tool to precisely simulate the performance of the designed sensor. In this study, the diaphragm performance was simulated to accurately predict the deflection vs. pressure distribution.

The maximum deflection of the diaphragm occurs at the center. Figs. 2 (b)-(f) show the obtained deflections caused in sensors 1, 2, 3, 4 and 5 when $75 \mathrm{~cm}$ of liquid is in place (full tank). The deflection values of each sensor under different pressures (and consequently under different heights of liquid) are shown in Table 1. At the end of section 2 some theoretical predictions of sensor behavior were provided. The calculated deflection caused by $75 \mathrm{~cm}$ of water was 4.1 $\mathrm{mm}$ from Eq. (2), which was similar to the deflection in the real device $(4.0 \pm 0.5 \mathrm{~mm})$, and considerably less than the $\sim 7.1 \mathrm{~mm}$ obtained by SolidWorks simulation. We attribute this difference to the modulus of the PMMA (around $3 \mathrm{GPa}$ ) being much greater than that of the silicone rubber $(0.0016 \mathrm{GPa})$. The much stiffer fiber will locally reinforce the more elastic diaphragm, restricting its elongation and reducing the strain in the region of the diaphragm close to the fiber. As the local maximum stresses occur in the center of the diaphragm, the von Mises equivalent stresses are shown in Fig. 2 (g) and are above the material strength value given by manufacturer (4.406 MPa from manufacturer and 5.6431 MPa from SolidWorks simulation), confirming that the diaphragm operational range observed experimentally (deflection of $4.0 \mathrm{~mm}$ ) is limited by the POFBG sensor's strain range.

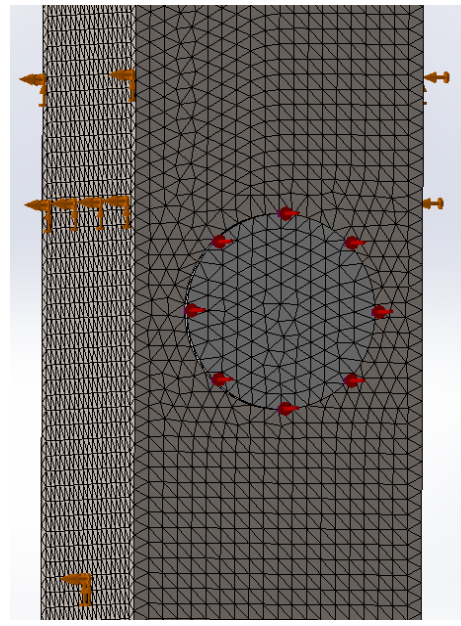

(a)

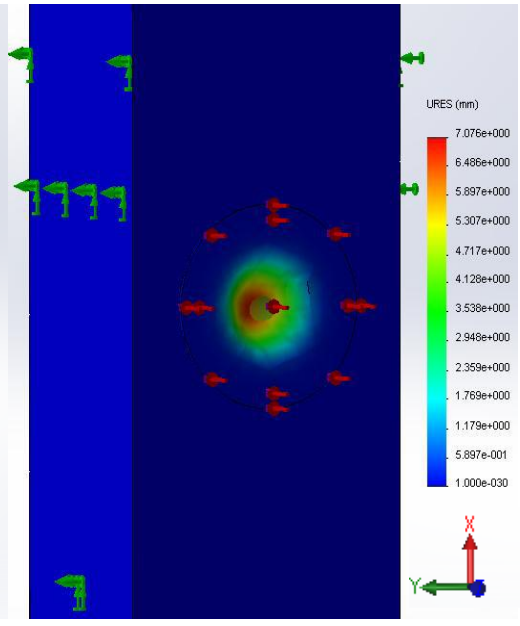

(b)

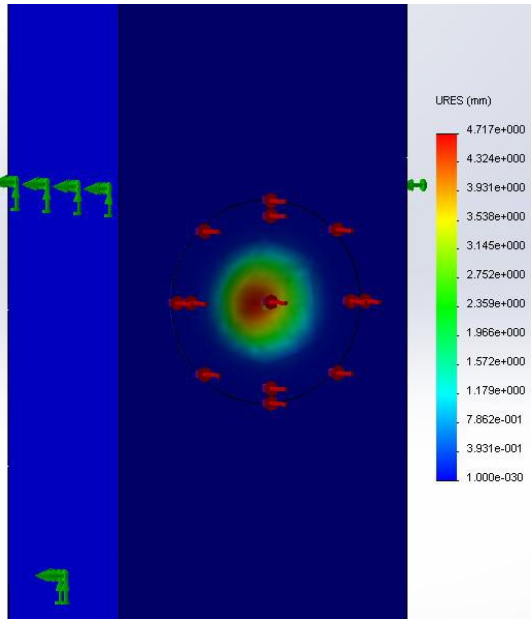

(c) 


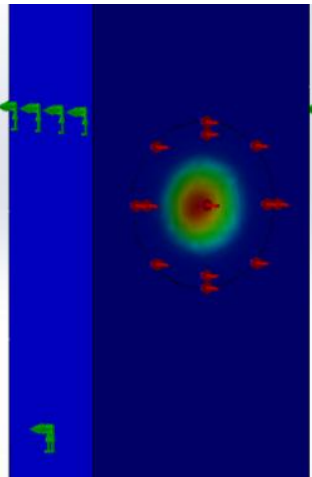

(d)
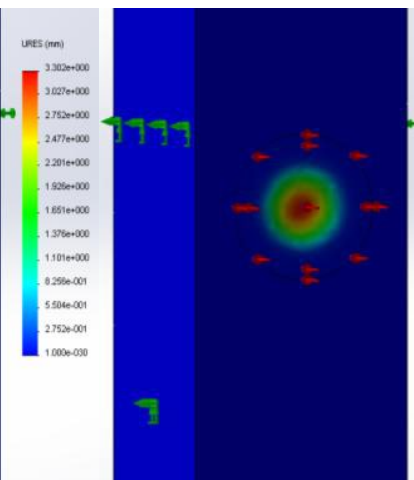

(e)

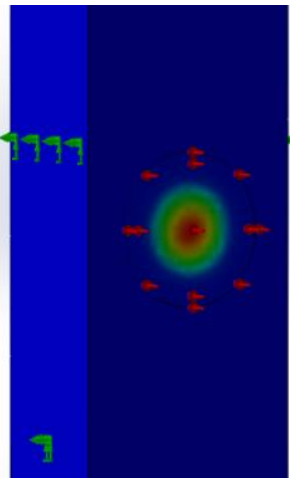

(f)

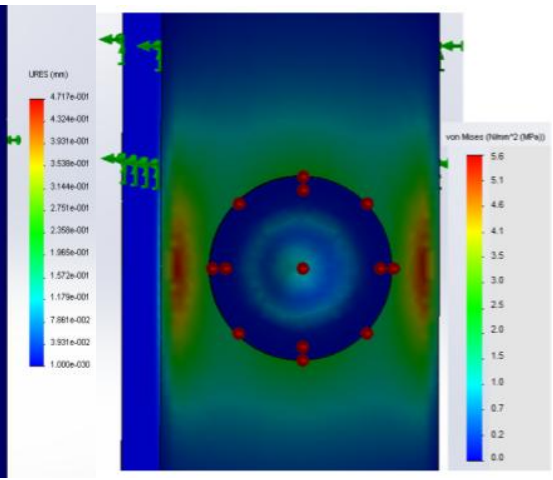

$(\mathrm{g})$

Fig. 2. 3D finite element model of the diaphragm/POFBG sensors system. (a) Meshed model. Simulated deflections caused in sensors

(b) 1, (c) 2, (d) 3, (e) 4 and (f) 5 when the tank is full (75 cm of water). (g) Von Mises stress distribution in the diaphragm.

Table 1. Simulated deflections in different sensors.

\begin{tabular}{cc}
\hline Sensor number / Pressure (Pa) & Deflection $(\mathbf{m m})$ \\
\hline Sensor 1 / 7339.4 & 7.076 \\
Sensor 2 / 4892.9 & 4.717 \\
Sensor 3 / 3425.1 & 3.302 \\
Sensor 4 / 1957.2 & 1.887 \\
Sensor 5 / 489.3 & 0.472 \\
\hline
\end{tabular}

\section{EXPERIMENTAL RESULTS}

The sensor performance was tested within a liquid level range of 0 to $75 \mathrm{~cm}$ and with a liquid level increment step of $5 \mathrm{~cm}$. Cyclic testing was performed to investigate the increasing and decreasing of liquid level. To guarantee the stability of the sensor, a constant liquid level was maintained for a period of 5 minutes at each step before the reading was taken. Less than $3 \mathrm{pm}$ shift of the central wavelength was observed over each such step period. The results of the configuration based on a single POFBG (Fig.1 (a)) are shown in Fig. 3 (a). The results demonstrate that the sensor gives a highly linear response over the entire measurement region. The wavelength shift induced over the $75 \mathrm{~cm}$ measurement region was around $4.3 \mathrm{~nm}$, leading to a mean sensitivity of $57.2 \pm 0.4 \mathrm{pm} / \mathrm{cm}$. Cyclic testing was also performed to investigate the repeatability of the sensor. They demonstrate good repeatability, and the overall sensitivity was found to be $57.3 \pm 0.4 \mathrm{pm} / \mathrm{cm}$.
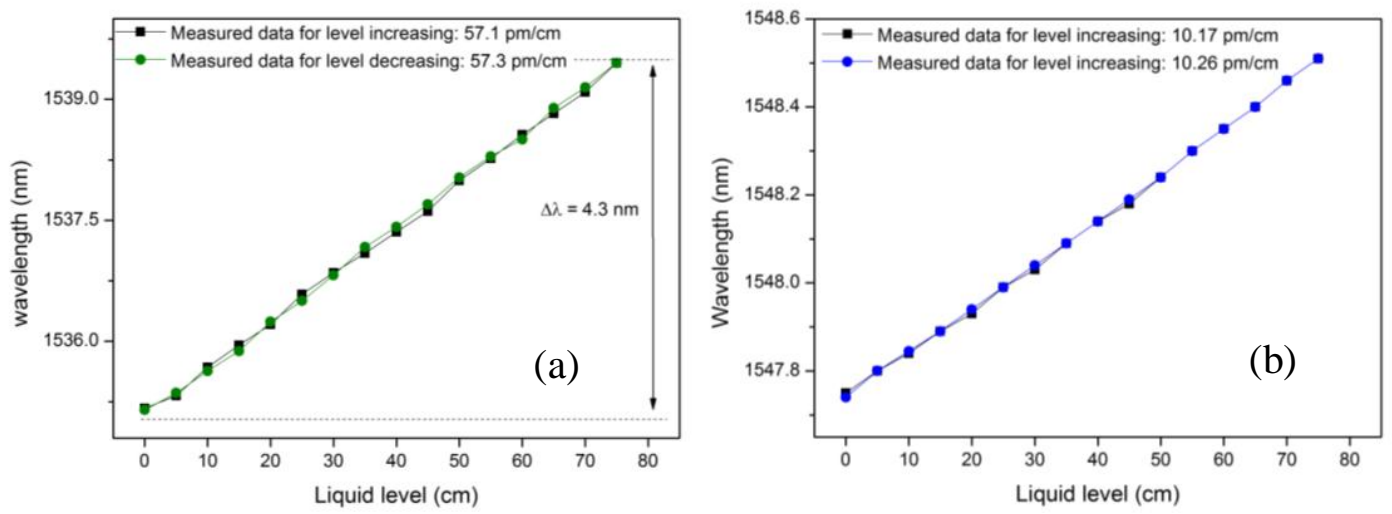

Fig. 3. (a) Response of the Bragg wavelength shift versus liquid level using a single (a) diaphragm/POFBG sensor and (b) diaphragm/FBG sensor based on a silica fiber. 
For comparison purposes, a similar sensor was fabricated using an FBG inscribed in $9 / 125 \mu \mathrm{m}$ silica optical fiber. Fig. 3 (b) shows the first cycle demonstrating that the sensor gives a much more repeatable response than the POFBG over the measurement range however with less sensitivity: $10.22 \pm 0.05 \mathrm{pm} / \mathrm{cm}$. The results of the five cycles demonstrate a consistent sensitivity with a mean value of $10.21 \pm 0.09 \mathrm{pm} / \mathrm{cm}$. These results indicate that the sensitivity using POFBGs is increased by more than 5 times, compared to the FBGs inscribed in silica fiber. Furthermore, it is considerably larger than that of the other previously published studies mentioned in the introduction [1-10].

The possibility of using a multi-POFBG based sensor was also explored. The experimental procedure was similar to the previous case. The sensor positions relative to the container base are shown in Fig. 1 (b). Here, we observed the behavior of each sensor when the liquid level was increased and decreased. Five full cycles were carried out. A set of optical fiber couplers was used in order to obtain the data from all the sensors simultaneously. Fig. 4 shows the first cycle of each of sensors 1, 2, 3 and 4. Sensor 1 measures the liquid level range from $0 \mathrm{~cm}$ to $75 \mathrm{~cm}$ (considering the $5 \mathrm{~cm}$ position shown in Fig. 2 as the beginning of the measurement range); sensor 2 from $20 \mathrm{~cm}$ to $75 \mathrm{~cm}$; sensor 3 from $35 \mathrm{~cm}$ to $75 \mathrm{~cm}$; and sensor 4 measures from $50 \mathrm{~cm}$ to $75 \mathrm{~cm}$. The wavelength shift was extracted and the sensitivity of each sensor was calculated, showing similar values for the increasing and decreasing of liquid level and a good linearity. The results showing a sensitivity with a mean value of $54.5 \pm 0.5 \mathrm{pm} / \mathrm{cm}, 54.0 \pm 0.7 \mathrm{pm} / \mathrm{cm}, 54.9 \pm 1.0 \mathrm{pm} / \mathrm{cm}$, and $53.7 \pm 1.9 \mathrm{pm} / \mathrm{cm}$ for the sensors 1,2, 3 and 4, respectively.
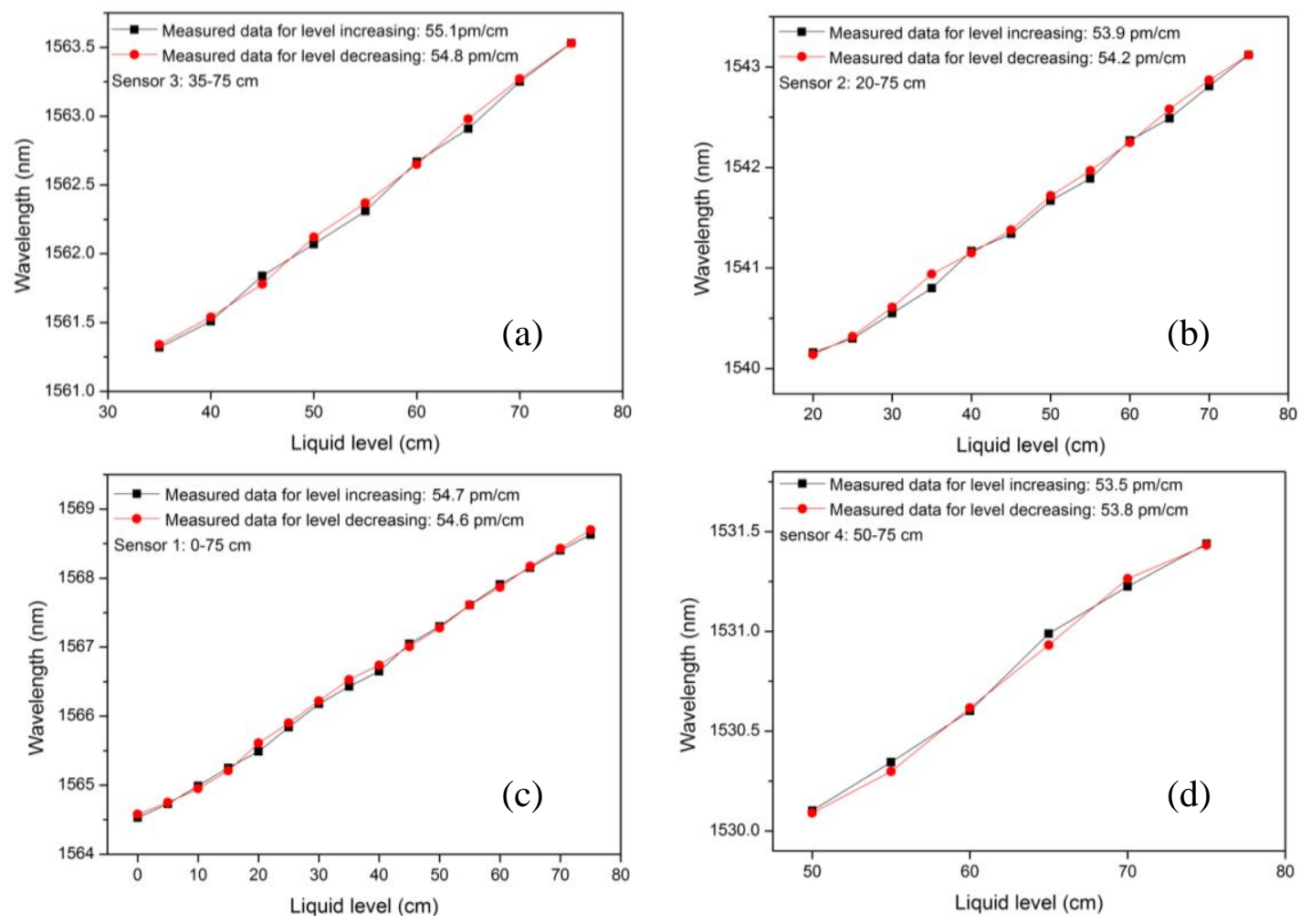

Fig. 4. Responses of the wavelength shift versus liquid level using multiple diaphragm/POFBG sensor: (a) sensor 1, (b) sensor 2, (c) sensor 3 and (d) sensor 4.

In addition, experiments were carried out to investigate the temperature response of the sensors. For this experiment four sensors (sensor 1, 2, 3 and 4) were submerged and sensor 5 was kept out of the liquid to compare the temperature behavior. The entire prototype was then placed in an environmental chamber (Sanyo Gallenkamp) under varying temperatures to study its response. The temperature was increased with steps of $5^{\circ} \mathrm{C}$ from $18^{\circ} \mathrm{C}$ up to $43^{\circ} \mathrm{C}$. In each step, the temperature was kept constant over 4 hours to ensure thermal equilibrium was achieved. Fig. 5 shows the measured wavelength shift of each sensor at different temperatures. From Fig. 5, the change in Bragg wavelength over the 25 
temperature variation was obtained for sensors 1 to 5 giving $-1.34 \mathrm{~nm},-1.35 \mathrm{~nm},-1.33 \mathrm{~nm},-1.30 \mathrm{~nm}$ and $-1.15 \mathrm{~nm}$, respectively.
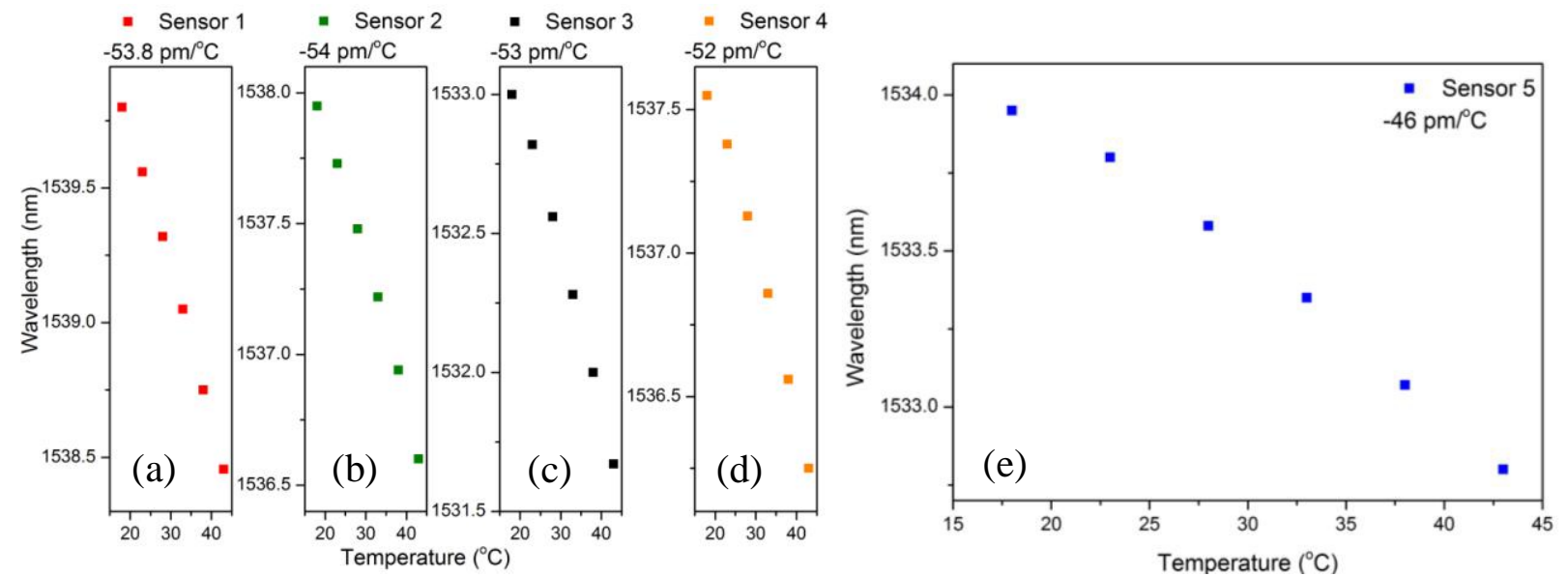

Fig. 5. Measured responses of the wavelength shift versus temperature variation for sensors submerged: (a) sensor 1, (b) sensor 2, (c) sensor 3, and (d) sensor 4. (e) No submerged sensor (sensor 5).

The measurement resolution of the individual sensor fabricated in this work was also analyzed. A crude method which is nevertheless used in the literature is to observe the short term fluctuations in the Bragg wavelength reading from the OSA and directly relate this to a liquid depth resolution using the measured sensitivity. In the case of the POFBG sensors the variation less than $3 \mathrm{pm}$ corresponds to roughly $2 \mathrm{~mm}$. A more rigorous approach is to undertake a statistical analysis based on least squares method of the results in Fig. 5, which leads to a resolution just under $10 \mathrm{~mm}$. The performance of the proposed sensor can be compared with some previous published studies referenced in 'Introduction' section: the sensors with larger measurement range have a lower resolution, while the sensors with higher resolution have a smaller range. Our results can be seen to compare very favorably in terms of range-to-resolution, which represents the number of effective measurement points.

\section{CONCLUSION}

For the first time a highly sensitive liquid level monitoring sensor based on a POFBG embedded in a silicone rubber diaphragm is designed and its performance investigated. A modeling analysis based on 3D finite element model of the diaphragm/POFBG sensors system is presented and discussed. The experimental results show that the proposed sensor has a high sensitivity to liquid level, great repeatability and exhibits a high linear response. The performance of an individual sensor was characterized and displays a high sensitivity $(54 \mathrm{pm} / \mathrm{cm})$, enhanced by more than a factor of 2 when compared to a sensor head configuration based on a silica FBG published in literature [20], resulting from the much lower elastic modulus of POF. Furthermore, the temperature behavior and measurement resolution were also studied in detail. This new configuration can be a useful tool in many different applications, such as aircraft fuel monitoring, biochemical and environmental sensing.

This work was supported by a Marie Curie Intra European Fellowship included in the 7th Framework Program of the European Union (POSSIBLE PIEF-GA-2013-628604 project).

\section{REFERENCES}

[1] G. Betta, L. Ippolito, A. Pietrosanto, and A. Scaglione, "Optical fiber-based technique for continuous-level sensing," IEEE Transactions on Instrumentation and Measurement 44, 686 (1995).

[2] G. Betta, A. Pietrosanto, and A. Scaglione, "A digital liquid level transducer based on optical fiber," IEEE Transactions on Instrumentation and Measurement 45, 551 (1996). 
[3] M. Lomer, A. Quintela, M. López-Amo, J. Zubia, and J. M. López-Higuera, "A quasi-distributed level sensor based on a bent side-polished plastic optical fiber cable," Measurement Science and Technology 18, 2261 (2007).

[4] C. Zhao, L. Ye, X. Yu, and J. Ge, "Continuous Fuel Level Sensor Based on Spiral Side-Emitting Optical Fiber," Journal of Control Science and Engineering, 2012, 267519 (2012).

[5] B. Yun, N. Chen, and Y. Cui, "Highly sensitive liquid-level sensor based on etched fiber bragg grating," IEEE Photon. Technol. Lett. 19, 1747 (2007).

[6] T. Guo, Q. D. Zhao, Q. Y. Dou, H. Zhang, L. F. Xue, G. L. Huang, and X. Y. Dong, "Temperature-insensitive fiber Bragg grating liquid-level sensor based on bending cantilever beam," IEEE Photon. Technol. Lett. 17, 2400 (2005).

[7] H. Y. Fu, X. W. Shu, A. P. Zhang, W. S. Liu, L. Zhang, S. L. He, and I. Bennion, "Implementation and characterization of liquid-level sensor based on a long-period fiber grating Mach-Zehnder interferometer," IEEE Sens. J. 11, 2878 (2011).

[8] C. B. Mou, K. M. Zhou, Z. J. Yan, H. Y. Fu, and L. Zhang, "Liquid level sensor based on an excessively tilted fiber grating," Opt. Commun. 305, 271 (2013).

[9] Q. Jiang, D. Hu, and M. Yang, "Simultaneous measurement of liquid level and surrounding refractive index using tilted fiber Bragg grating," Sensors and Actuators A 170, 62 (2011).

[10]B. Gu, W. Qi, Y. Zhou, Z. Wu, P. P. Shum, and F. Luan, "Reflective liquid level sensor based on modes conversion in thin-core fiber incorporating tilted fiber Bragg grating," Opt. Express 22, 11834 (2014).

[11] W. Yuan, L. Khan, D. J. Webb, K. Kalli, H. K. Rasmussen, A. Stefani, and O. Bang, "Humidity insensitive TOPAS polymer fiber Bragg grating sensor," Opt. Express 19, 19731 (2011).

[12] C. C. Ye, J. M. Dulieu-Barton, D. J. Webb, C. Zhang, G. D. Peng, A. R. Chambers, F. J. Lennard, and D. D. Eastop, "Applications of polymer optical fiber grating sensors to condition monitoring of textiles," J. Phys. Conf. Ser. 178, 012020 (2009).

[13] W. Zhang, D. J. Webb, and G.-D. Peng, "Polymer optical fiber Bragg grating acting as an intrinsic biochemical concentration sensor," Opt. Lett. 37, 1370 (2012).

[14] W. Zhang, and D. J. Webb, "Humidity responsivity of poly(methyl methacrylate)-based optical fiber Bragg grating sensors," Opt. Lett. 39, 3026-3029 (2014).

[15] J. Huang, L. Xinwei, W. Hanzheng, Y. Lei, W. Tao, G. Zhan, and X. Hai, "Polymer optical fiber for large strain measurement based on multimode interference," Opt. Lett., 37, 4308 (2012).

[16] M. F. Ashby, "Materials selection in mechanical design - 2nd edition", Oxford, UK, 389 (1999).

[17]A. Othonos, and K. Kalli, "Fiber Bragg Gratings - Fundamentals and Applications in Telecommunications and Sensing", Artech House (1999).

[18] http://www.dowcorning.com/content/rubber/

[19]H. Y. Liu, G. D. Peng, and P. L. Chu, "Polymer fiber Bragg gratings with 28-dB transmission rejection", IEEE Photon. Technol. Lett. 14, 935 (2002).

[20]D. Sengupta, and P. Kishore, "Continuous liquid level monitoring sensor system using fiber Bragg grating," Opt. Eng. 53, 017102 (2014). 